\title{
ORDER MATTERS WHEN CHOOSING SETS
}

\author{
WARREN B. MOORS AND JULIA C. NOVAK
}

Abstract. Given natural numbers $t, w$ and $v$ we show, using high school algebra, that if $1 \leqslant w \leqslant t<v$ then $((v \operatorname{ch} t) \operatorname{ch} w) \leqslant((v \operatorname{ch} w) \operatorname{ch} t)$. Here we denote " $n$ choose $r$ " by $(n \operatorname{ch} r)$.

Mathematics subject classification (2010): Primary 05A20; Secondary 05A05, 94A60.

Keywords and phrases: Key distribution patterns, combinatorial inequalities, cryptography.

\section{REFERENCES}

[1] Solomon W. Golomb, Iterated Binomial Coefficients, Amer. Math. Monthly, 87 (1980), 719-727.

[2] Julia C. Novak, Generalised Key Distribution Patterns, PhD Thesis, Royal Holloway, University of London, (in preparation). 